\title{
Optoelectronic properties of CuPc thin films deposited at different substrate temperatures
}

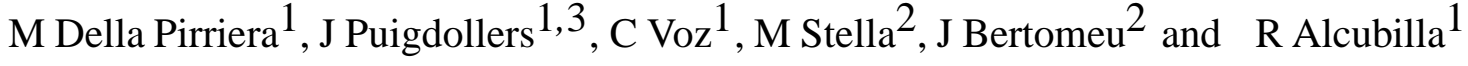 \\ 1 Micro and Nanotechnology Group. Dept. Enginyeria Electrònica, Universitat Politècnica Catalunya, \\ 08034 Barcelona, Spain \\ 2 Dep. Física Aplicada i Òptica. Universitat de Barcelona, Spain \\ 3 Author to whom any correspondence should be addressed.
}

\begin{abstract}
Structural and optical characterization of copper phthalocyanine thin film thermally deposited at different substrate temperatures was the aim of this work. The morphology of the films shows strong dependence on temperature, as can be observed by atomic force microscopy and $\mathrm{x}$-ray diffraction spectroscopy, specifically in the grain size and features of the grains. The increase in the crystal phase with substrate temperature is shown by x-ray diffractometry. Optical absorption coefficient measured by photothermal deflection spectroscopy and optical transmittance reveal a weak dependence on the substrate temperature. Besides, the electro-optical response measured by the external quantum efficiency of Schottky ITO/CuPc/Al diodes shows an optimized response for samples deposited at a substrate temperature of $60^{\circ} \mathrm{C}$, in correspondence to the $I-V$ diode characteristics.
\end{abstract}




\section{Introduction}

Organic semiconductors have been proposed for successful application in optical and electronic devices. In the case of solar cells, the use of organic semiconductors allows the fabrication of large area devices at relatively low cost and on flexible substrates. Two technological approaches are currently studied. In the case of polymer materials, blended donor and acceptor in solution are used to fabricate the device by spin-coating processes, but for small molecular weight organic material the layers of the device are produced by thermal evaporation. Energy conversion efficiency has been gradually increased in recent years and laboratory efficiencies higher than $6 \%$ are currently reported both for polymer and smallmolecule solar cells [1].

Power conversion in an organic solar cell is composed of three processes, exciton generation by optical absorption, exciton dissociation into carriers and, finally, carrier collection at the electrodes. In spite of the extremely efficient exciton dissociation occurring at the adjacent donor and acceptor sites, only a small number of excitons generated near the donor-acceptor interface contribute to the photocurrent. Low charge carrier mobilities and recombination of photogenerated carriers are responsible for the low photocurrent obtained [2]. Several studies are addressed to control the morphology of the films in order to optimize electrical transport and suppress exciton recombination. The morphology can be improved in terms of charge transport properties by optimizing deposition conditions, such as substrate temperature and growth rates.

Although copper phthalocyanine $(\mathrm{CuPc})$ is one of the most studied p-type organic semiconductors, few studies have addressed its molecular orientation and structure as a function of the substrate temperature. Depending on the deposition conditions different molecular orientations and crystalline structures can be obtained. CuPc molecules have a planar structure (figure 1(a)), and in a crystal they are packed together forming column-like structures (figure 1(b)). Two typical crystalline polymorphous structures are usually observed in CuPc material: the well-known metastable $\alpha$ - and stable $\beta$-phases [3]. The main differences between them are the molecular overlapping area (figure 1(c)). Generally, powder $\mathrm{CuPc}$ shows the so-called ' $\beta$-phase' $\left(a=19.4 \AA, b=4.8 \AA, c=14.6 \AA, \beta=120^{\circ}\right)$. For $\mathrm{CuPc}$ thin films 
deposited on substrate at room temperature, the film grows in the $\alpha$-phase ( $a=25.92$ $\AA, b=3.79 \AA, c=23.92 \AA, \beta=90.4^{\circ}$ ), whereas the $\beta$-phase is obtained for samples deposited or annealed at higher temperature. Because of the anisotropy on the stacked $\mathrm{CuPc}$ molecules, two main types of molecular orientation are usually obtained. Both orientations are characterized by a herringbone structure. In one type, the molecules' orientation is in the $\left[\begin{array}{lll}0 & 1 & 0\end{array}\right]$ direction, normal to the substrate surface, whereas in the other one, molecules are lying perpendicular to the substrate surface ([ $\left[\begin{array}{lll}0 & 1 & 0\end{array}\right]$ direction parallel to the surface). Since carriers (electrons and holes) move easily along the [0 $10]$ direction, differences in the molecule orientation have a dramatic effect on the charge transport properties and on the final electrical performance of the device [4].

In this paper the optical and structural properties of copper phthalocyanine $(\mathrm{CuPc})$ thin films deposited at different substrate temperatures will be presented. Optical properties were evaluated by absorbance and photothermal deflection spectroscopy (PDS) measurements. Atomic force microscopy (AFM) and x-ray diffraction spectroscopy were used to study the dependence of the crystalline structure as a function of the substrate temperature. The external quantum efficiency (EQE) was also measured for Schottky devices.

\section{Experimental}

$\mathrm{CuPc}$ films were deposited by thermal evaporation in a high vacuum chamber with a base pressure of $10^{-6}$ mbar at different substrate temperatures $(30,60,90$ and $\left.120^{\circ} \mathrm{C}\right)$. The materials are commercially available from Sigma Aldrich, with $99 \%$ purity, with no further purification process. The deposition rate was $1 \AA / \mathrm{seg}$. for a sample thickness of $700 \mathrm{~nm}$ as measured by a surface profiler (Dektak Veeco). Structural properties of the films were evaluated by x-ray diffraction (Siemens D500 XRD system) and atomic force microscopy (Multimode Veeco AFM system). XRD was used to evaluate the crystallinity of the samples whereas AFM was used to analyse the surface morphology. The optical absorption coefficient of the films was determined by PDS and optical absorption measurements. In the PDS technique, widely used for inorganic amorphous and nanostructured semiconductors, the heat created by nonradiative recombination of absorbed photons is measured with a very high sensitivity. 
The PDS technique allows us to measure low-level absorption features, revealing defect induced sub-gap absorptions. Since these sub-band gap absorption features are typically characterized by very low absorption coefficients, it is not possible to resolve them using common transmission and reflection measurements. To get more information about the electro-optical response of $\mathrm{CuPc}$ thin films, the EQE of Schottky devices was also measured. The structure of the fabricated Schottky diodes was glass/ITO/CuPc/Al. ITO coated glasses were cleaned by ultraviolet ozone treatment. The ITO was introduced into the organic semiconductor evaporator in order to deposit the organic thin film. After $700 \mathrm{~nm}$ of $\mathrm{CuPc}$, the sample was transported to the metal evaporator in air (i.e. exposed to ambient oxygen). Aluminium electrodes (100 $\mathrm{nm}$ thick and $1 \mathrm{~mm}^{2}$ area) were thermally evaporated through a shadow mask. The final devices were not encapsulated and were characterized in air (i.e. exposed to ambient oxygen). The EQE was measured using a xenon lamp (400 W) as light source, being dispersed by a Jobin Yvon 180 triax monochromator and mechanically chopped at low frequencies $(<100 \mathrm{~Hz})$. A digital lock-in amplifier (Stanford Research 830) was used to obtain the photocurrent. The current-voltage characteristics of the devices were measured using a parameter analyzer (model HP4156 from Agilent).

\section{Results and discussion}

Figure 2 shows the x-ray diffraction patterns of the films (700 nm thick) deposited at different substrate temperatures on glass. The background due to scattering from the amorphous glass substrate has been subtracted. All the patterns present the characteristic features of the $\mathrm{CuPc}$ polymorphous structure characterized by a single

sharp reflection at $2 \theta$ of $6.9^{\circ}(d=12.9 \AA)$. This peak arises from the interlayer spacing of stacks of tilted molecules. The single peak is the result of diffraction from the $\left(\begin{array}{lll}2 & 0 & 0\end{array}\right)$ lattice planes separated by approximately the inter-stacking distances, and, therefore, implies that the trace of the herringbone pattern is parallel to the substrate. This distance between the plane of the copper atoms in one layer and that in the next layer is in fair agreement with the reference values $(12.6 \AA)$ [5, 6].

The peak position is the result of the $\alpha$ form structure of the CuPc crystals. These results are in agreement with other works that have shown that the $\beta$ structure is 
obtained if the $\mathrm{CuPc}$ is grown at a temperature of over $200^{\circ} \mathrm{C}[7,8]$. This peak at $2 \theta=$ $6.83^{\circ}$ gives an interplanar distance of $d\left(\begin{array}{lll}2 & 0 & 0\end{array}\right)=14.1 \AA$. It can be seen that the intensity of the $(200)$ peak increases for increasing substrate temperatures, so this indicates that the crystal phase increases instead of the amorphous phase. The position of the peak is constant for the different substrate temperatures, so the layers have a crystal phase with the $\alpha$ form structure. In contrast to other works [9], other peaks are present in figure 2, such as (400) and (313), corresponding to the monoclinic structure.

The domain size of the crystalline grains can be calculated from the broadening of the diffraction pattern by using the Scherrer formula [6]. In our samples, the calculated crystallite sizes are around $(40 \pm 2) \mathrm{nm}$ for the sample deposited at $30^{\circ} \mathrm{C}$, $(38 \pm 2) \mathrm{nm}$ for $60^{\circ} \mathrm{C},(100 \pm 2) \mathrm{nm}$ for $90^{\circ} \mathrm{C}$ and $(130 \pm 2) \mathrm{nm}$ for $120^{\circ} \mathrm{C}$. The crystalline grains increase with substrate temperature [9]. Crystallite sizes are in agreement with the reported values for $\mathrm{CuPc}$ thin films deposited at room temperature [10].

In order to get more information on the structure of the deposited films, the samples were examined using an atomic force microscope. Figure 3 shows AFM images of the $\mathrm{CuPc}$ films deposited at different substrate temperatures, with different scales. At room temperature can be seen a mix of granular (around $100 \mathrm{~nm}$ of size) and needle-like features $\left(\sim 50 \times 250 \mathrm{~nm}^{2}\right)$, but at $60^{\circ} \mathrm{C}$ substrate temperature the surface morphology is characterized by needle-like features $\left(\sim 50 \times 625 \mathrm{~nm}^{2}\right)$ more oriented in the film plane, with a length and aspect ratio (length/width) depending on the substrate temperature. The size of these features increases with the temperature. Planar terraces on the needles are also visible. The elongated grains along the molecular stack direction are due to the face-to-face intermolecular adhesion of the CuPc molecules. At $90^{\circ} \mathrm{C}$ the needles are more disoriented, transforming into rod-like crystals which can be seen at $120^{\circ} \mathrm{C}$.

Regarding the optical properties of the deposited films, in figure 4 we show the optical absorption coefficient $(\alpha)$ of CuPc films, deposited at different substrate temperatures, as a function of the incident photon energy. PDS is a very sensitive technique that makes it possible to measure very low-level absorptions in the sub-gap 
region, but the measurements must be calibrated using transmittance data that are also more reliable in the high-level absorption region of the spectrum. As a result, the spectra are composed of data calculated from transmittance measurements ( $h v>1.7$ $\mathrm{eV})$ and data calculated from PDS measurements $(h v<1.7 \mathrm{eV})$. Two absorption bands, centred at $2 \mathrm{eV}$ and $3.5 \mathrm{eV}$, respectively, are observed in the band-to-band transitions region. Such features correspond to the known $Q$ and Soret bands $[11,12]$. The exponential decrease that is situated at energy values between 1.7 and $1.5 \mathrm{eV}$ indicates the presence of band tails, similarly to the case of amorphous silicon. The absorption coefficient in the sub-gap region is probably determined by the presence of localized energy states associated with defects in the material. The most relevant feature in this region is the presence of four absorption peaks situated at $1.04 \mathrm{eV}, 1.12 \mathrm{eV}$, $1.20 \mathrm{eV}$ and $1.33 \mathrm{eV}$, respectively. The nature of such absorption phenomena has already been discussed in a previous work [13].

A slight effect of the substrate temperature is registered on the absorption coefficient. For energy values lower than $1 \mathrm{eV}$ the absorption grows with the deposition temperature. This result indicates that probably the sample deposited at the highest temperature is affected by the highest defect density. The direct dependence of the absorption level on the temperature is not repeated in other parts of the spectra, such as around 1.25, 2 and $3 \mathrm{eV}$, where the highest absorption values are registered for the sample deposited with $90^{\circ} \mathrm{C}$.

The optical gap of the CuPc samples has been estimated by performing a fit to the exponential part of the spectra using the equation for allowed direct transitions [14-16]:

$$
\alpha(h v)=A \cdot(h v-E o g)^{1 / 2}
$$

where $A$ is a constant and $E_{0 g}$ the optical gap of the semiconductor. The fit, calculated from the plot of $\alpha^{2}$ versus $h v$, is shown in figure 5. The values obtained for all the samples are around $1.62 \mathrm{eV}$ and no important change is observed in the function of the substrate temperature.

The transmittance spectrum of the CuPc is shown in the inset of figure 5. This spectrum is independent of the substrate temperature. The samples present similar absorption behaviour in the visible region of the spectra, characterized by two distinct absorption bands located around $2 \mathrm{eV}$ (Q-band) and $3.5 \mathrm{eV}$ (B- or Soret band). These bands are assigned to the $\pi \rightarrow \pi *$ transitions of the macrocyclic $\pi$ - system [11, 
17]. The two peaks observed in the near-infrared spectra are characteristic of the metal absorption bands of the phthalocyanines (copper in our case).

To evaluate the properties of $\mathrm{CuPc}$ thin films for their use in organic devices, a series of Schottky diodes were fabricated at different substrate temperatures. The structure was glass/ITO/CuPc/Al. In figure 6 the photovoltaic parameters such as $J_{\mathrm{SC}}$, $V_{\mathrm{OC}}$ and FF show a better response for the device with $\mathrm{CuPc}$ thin film deposited at $60^{\circ} \mathrm{C}$. The curves of the EQE for the different fabricated diodes are shown in figure 7. All the samples show similar behaviour. The strong reduction in EQE for wavelengths below 300 $\mathrm{nm}$ (see figure 5) is not related to the CuPc film, but due to the absorption of the front ITO electrode.

The EQE follows the absorbance in the long wavelength range $(>600 \mathrm{~nm})$, which is known as symbatic behaviour. In contrast, the behaviour becomes antibatic for shorter wavelengths, when the optical absorbance is lower.

In organic semiconductors, the absorption of photons generates excitons, rather than free carriers. These excitons should be dissociated into charge carriers (polarons) in order to contribute to the photocurrent. It is well accepted that excitons are dissociated only within narrow photoactive regions close to the electrodes [18-20]. Then, the main contribution to the photocurrent will come from excitons dissociated at the electrode which offers the shortest distance for the less mobile carrier (electrons for the p-type $\mathrm{CuPc}$ ). In ITO/CuPc/Al structures, electrons should be collected at the Al contact (cathode), whereas holes at the ITO anode contribute to the photocurrent. Since the CuPc Schottky diodes are illuminated from the front ITO electrode, antibatic behaviour will be expected for excitons generated near the front contact, whereas symbatic behaviour would be expected for excitons generated near the top $\mathrm{Al}$ cathode. The higher response observed for devices deposited at $60{ }^{\circ} \mathrm{C}$ is probably due to the high crystalline fraction together with the low density of states obtained for these films as stated from XRD and PDS measurements.

\section{Conclusions}

Copper phthalocyanine thin films deposited at different sub- strate temperatures show polycrystalline structure. Increas- ing substrate temperature allows growing films with higher crystalline fraction, as observed by XRD and AFM measure- ments. 
However, no significant changes are observed in the optical properties. The films present high optical absorp- tion coefficients, higher than $5 \times 10^{-5} \mathrm{~cm}^{-1}$. An exponential decrease in the optical absorption, at energy values between 1.7 and 1.5 $\mathrm{eV}$, indicates the presence of band tails, similarly to the case of microcrystalline inorganic semiconductors. The absorption coefficient in the sub-gap region is very low and shows a slight dependence on the substrate temperature. This sub-gap absorption is probably due to the presence of localized energy states associated with defects in the material. The aim of this work is to have deeper knowledge about how one can improve the conductivity in the CuPc layer by varying the deposition parameters. We made Schottky diodes in order to know which are the optimal deposition parameters to increase the hole transport in CuPc. The EQE of the fabricated Schottky diodes show that at a substrate temperature of $60{ }^{\circ} \mathrm{C}$ the CuPc Schottky diodes present the best optoelectronic response.

\section{Acknowledgments}

The authors acknowledge the financial support from the following programmes of the Spanish government: TEC- 02716 and HOPE CSD2007-00007 (Consolider-Ingenio 2010). They also appreciate the financial collaboration from the XaRMAE of the Generalitat of Catalunya. 


\section{References}

[1] Kim J Y, Lee K, Coates N E, Moses D, Nguyen T-Q, Dante M and Heeger A J 2007 Science 317222

[2] Sun S and Sariciftci N 2005 Organic Photovoltaic Mechanisms, Materials and Devices (London: Taylor and Francis)

[3] Berger O, Fischer W J, Adolphi B, Tierbach S, Melev V and Schreiber J $2000 \mathrm{~J}$. Mater. Sci. Mater. Electron. 11331

[4] Yamashita Y, Suzuki H, Kojima N, Ohshita Y and Yamaguchi M 2006 Photovoltaics Energy Conversion, Conf. Record of the 2006 IEEE 4th World Conf. (Hawaii)

[5] Komiyama M, Sakakibara M and Hiorai H 1987 Thin Solid Films 151 L109

[6] Cullity B D 1977 Elements of X-ray Diffraction (Boston, MA: Addison-Wesley)

[7] Nonaka T, Nakagawa Y, Mori Y, Hirai M, Matsunobe T, Nakamura M, Takahagi T, Ishitani A, Lin H and Koumoto K 1995 Thin Solid Films 256262

[8] Lozzi L, Santucci S, La Rosa S, Delley B and Picozzi S 2004 J. Chem. Phys. 121 1883

[9] Bao Z, Lovinger A J and Dodabalapur A 1996 Appl. Phys. Lett. 693066

[10] Xiao K, Liu Y, Yu G and Zhu D 2003 Appl. Phys. A 77367

[11] Ritz A and Lüth H 1983 Appl. Phys. A 3175

[12] Edwards L and Gouterman M 1970 J. Mol. Spectrosc. 33292

[13] Stella M, Voz C, Puigdollers J, Rojas F, Fonrodona M, Escarré J, Asensi J M, Bertomeu J and Andreu J 2006 J. Non-Crystal. Solids 3521663

[14] El-Nahass M M, Bahabri F S and Al-Harbi R 2001 Egypt. J. Solids 2411

[15] Varghese S, Iype M, Mathew E J and Menon C S 2002 Mater. Lett. 561078

[16] Shalimova K V 1975 Semiconductors Physics (Paris: Mir)

[17] Schechtman B H and Spicer W E 1970 J. Mol. Spectrosc. 3328

[18] Ghosh A K and Feng T 1978 J. Appl. Phys. 495982

[19] Harrison M G, Grüner J and Spencer G C W 1997 Phys. Rev. B 557831

[20] Voz C, Puigdollers J, Martin I, Muñoz D, Orpella A, Vetter M and Alcubilla R 2005 Sol. Energy Mater. Sol. Cells 87567 
Figure 1. (a) Scheme of the molecular structure of CuPc, (b) CuPc stacked molecules and (c) $\alpha$ and $\beta$ CuPc structures.

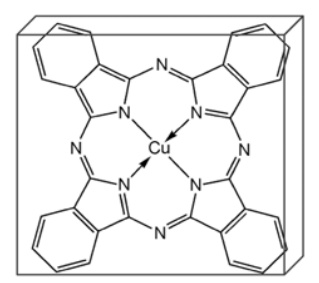

(a)

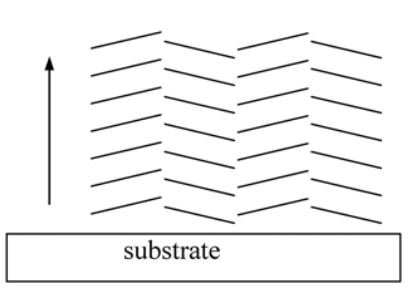

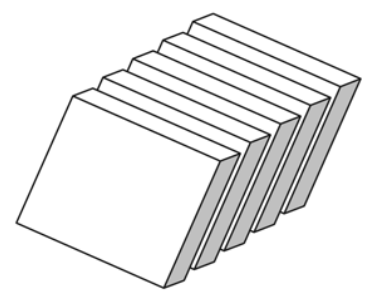

(b)

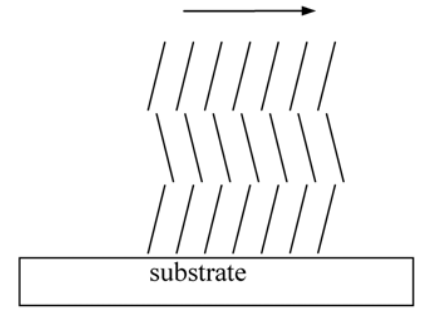

(c) 
Figure 2. XRD spectra of the CuPc layer deposited at different substrate temperatures.

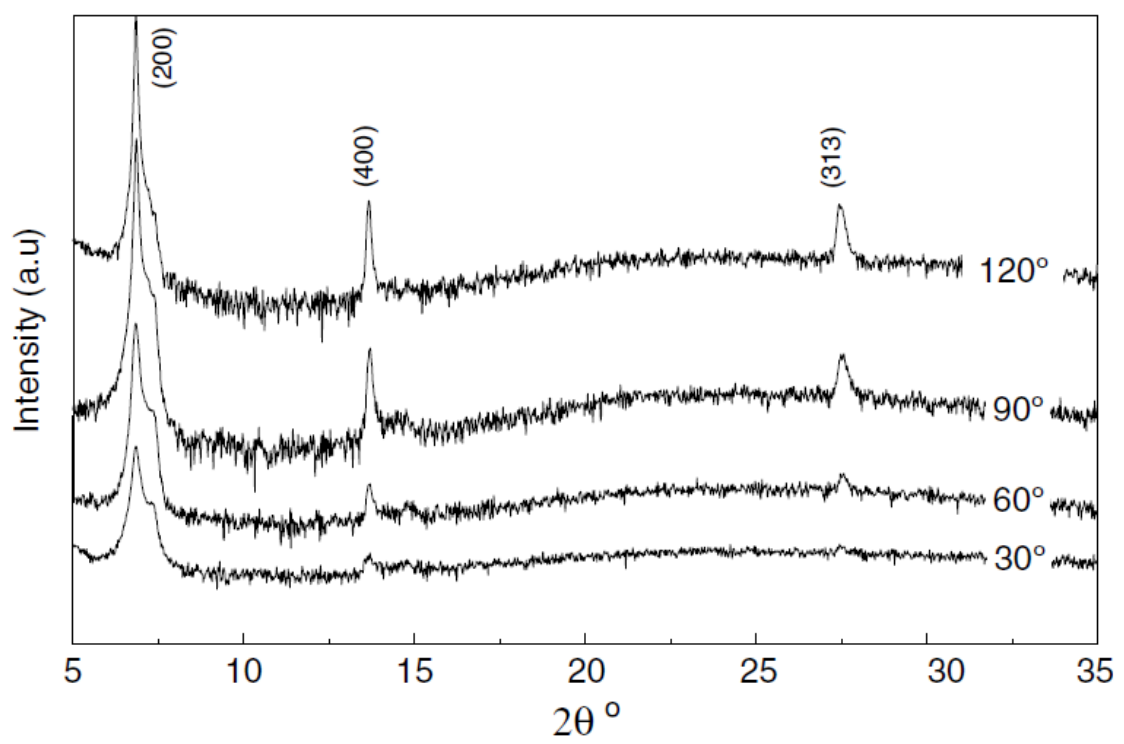


Figure 3. AFM images for $700 \mathrm{~nm} \mathrm{CuPc}$ layers deposited on bare glass at different substrate temperatures, from right to left: $30,60,90$ and $120^{\circ} \mathrm{C}$ at different scales $(5 \mu \mathrm{m}$, upper images and $500 \mathrm{~nm}$, lower images).

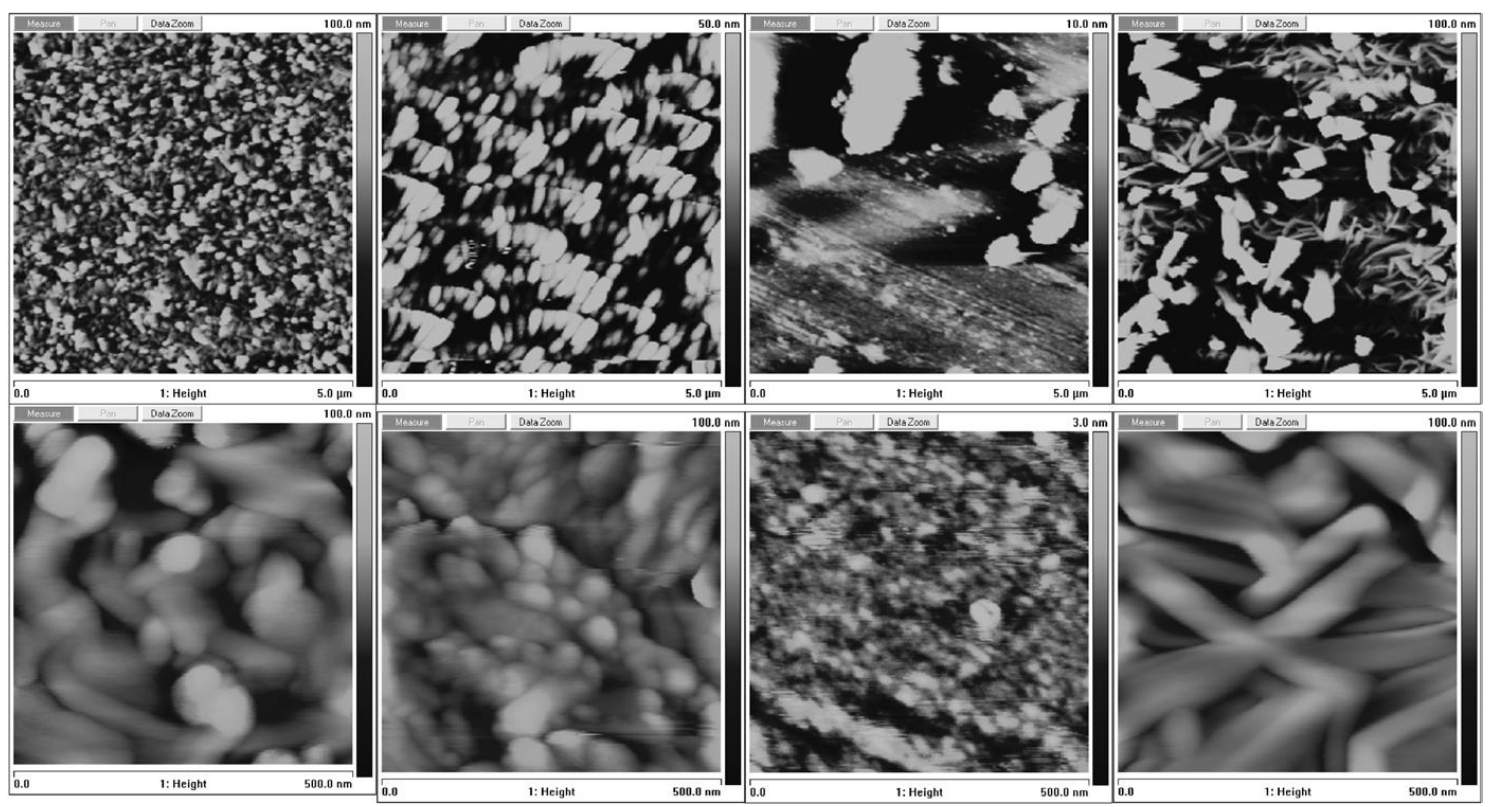


Figure 4. Absorption coefficient measurements of $\mathrm{CuPc}$ at different substrate temperatures.

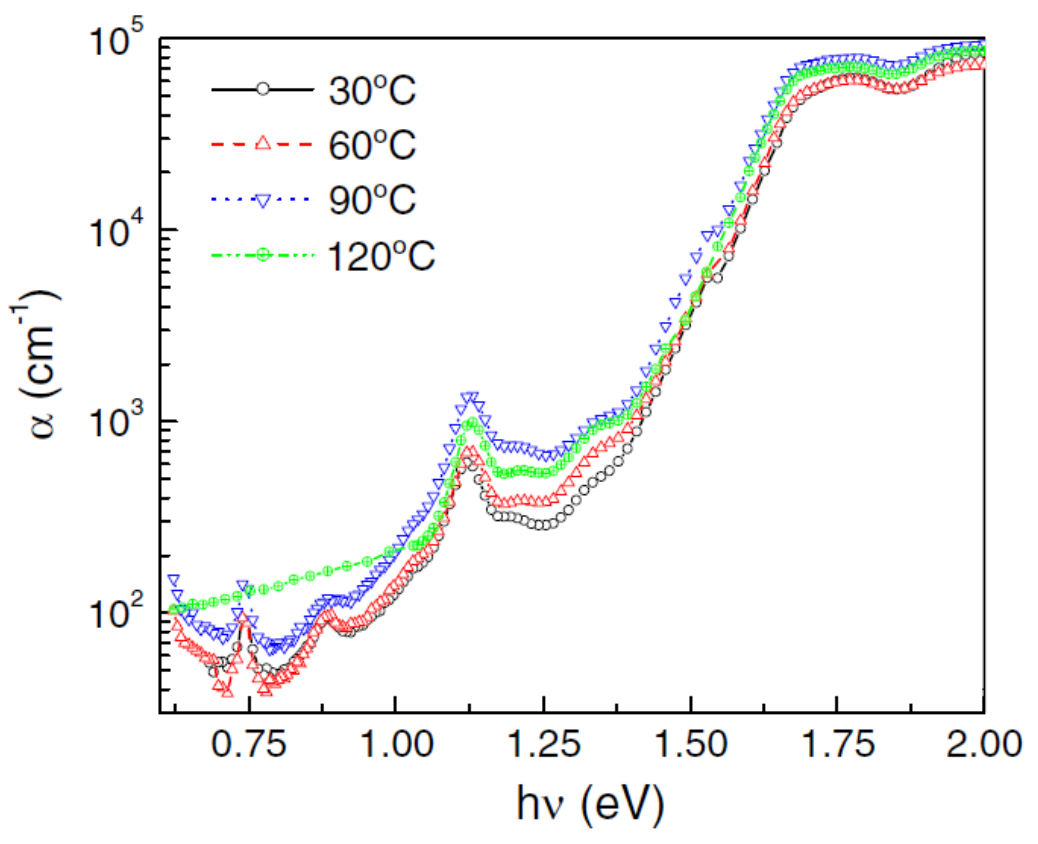


Figure 5. Plot of $\alpha^{2}$ versus energy and calculation of the optical gap for the sample deposited at $30^{\circ} \mathrm{C}$. Inset: optical transmittance of the CuPc thin-film layer deposited at $30^{\circ} \mathrm{C}$.

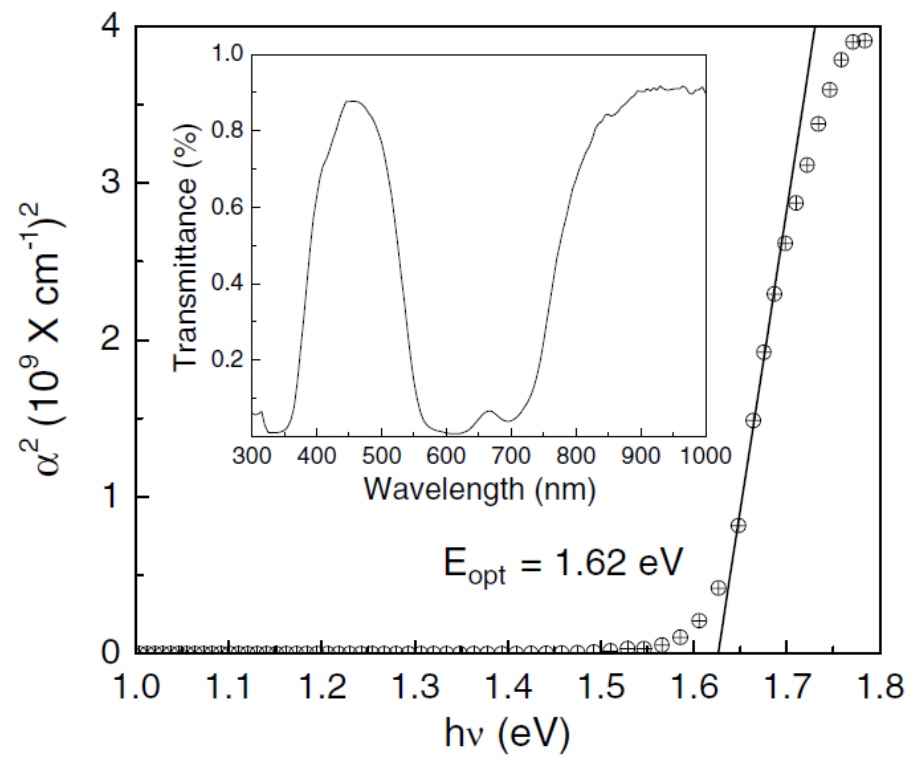


Figure 6. Electrical characteristics of Schottky device of CuPc deposited at different substrate temperatures.

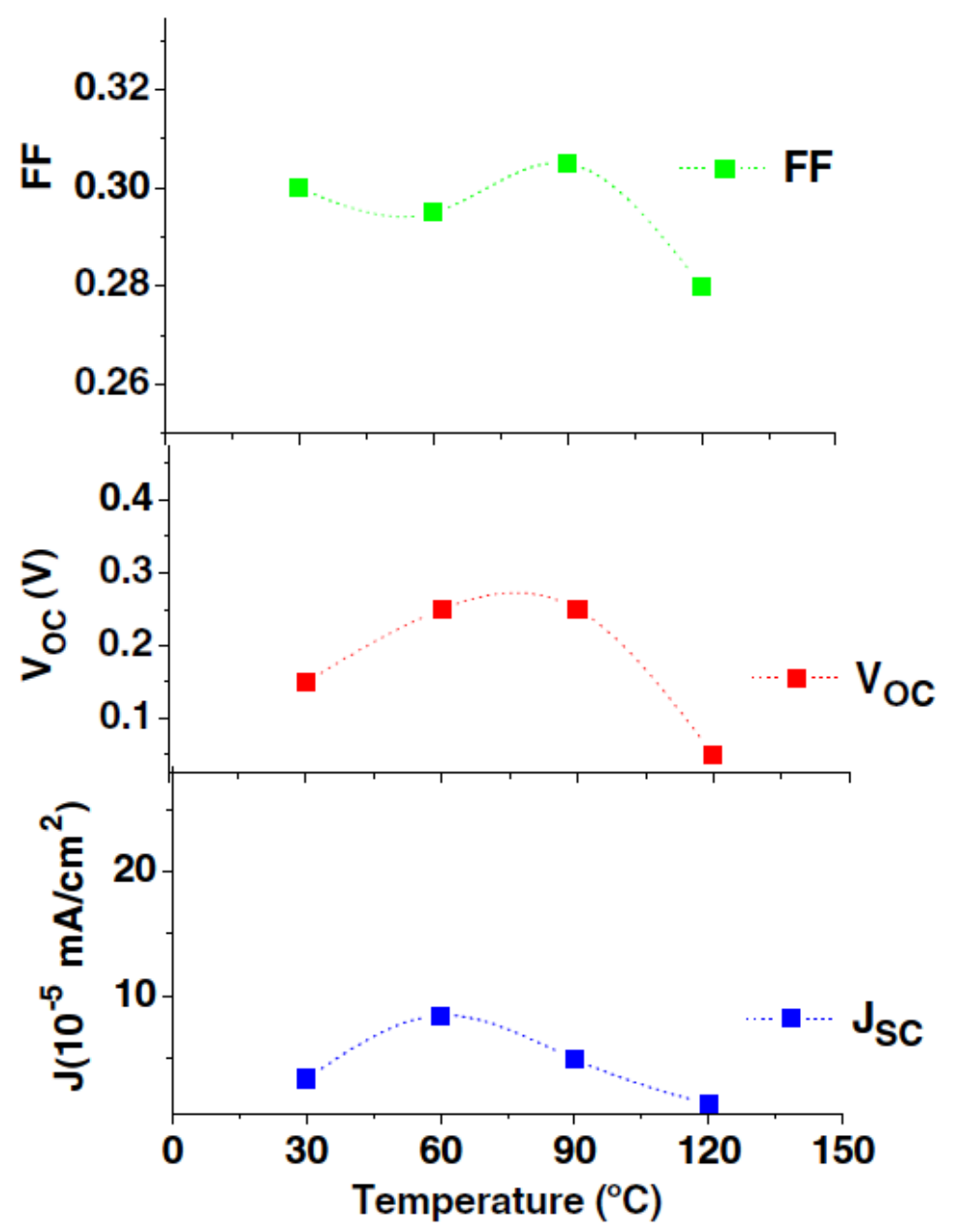


Figure 7. The EQE of the Schottky diodes (ITO/CuPc/Al) for devices with CuPc thinfilm layer deposited at different substrate temperatures $\left(30,60,90\right.$ and $\left.120^{\circ} \mathrm{C}\right)$.

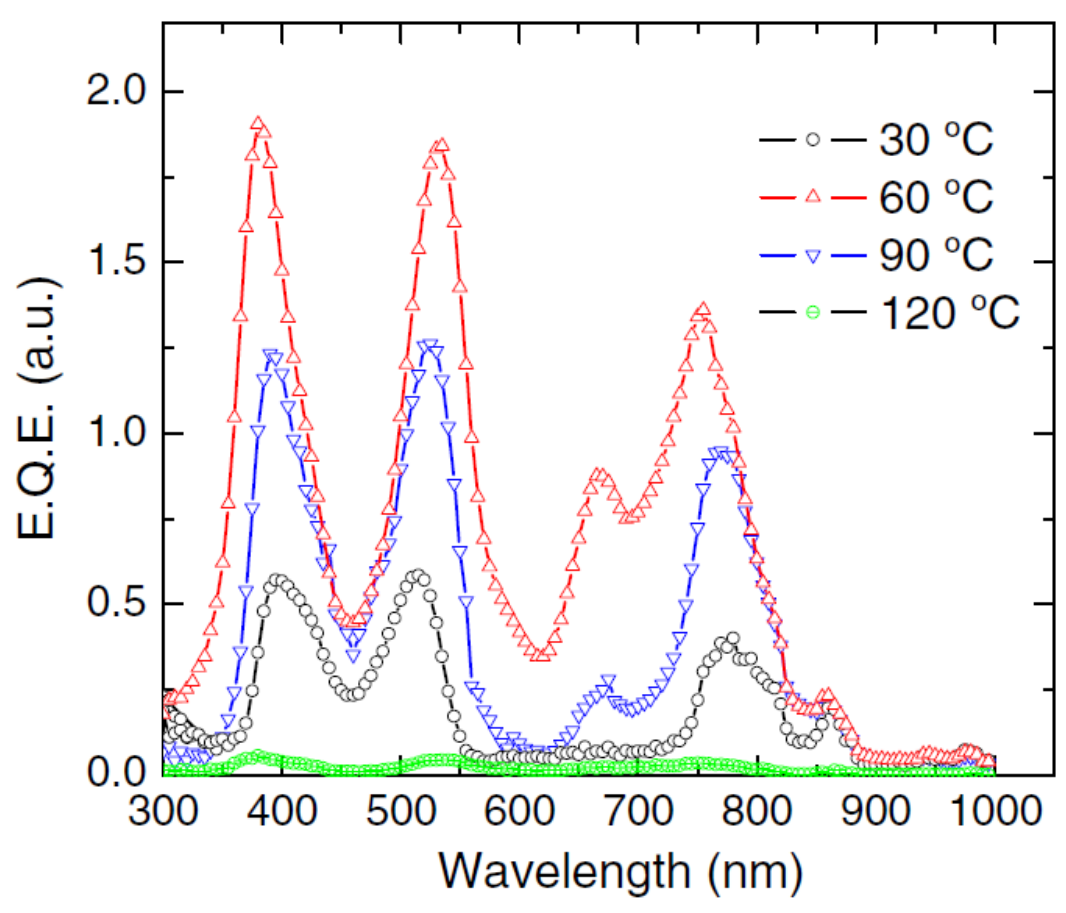

